\title{
Imputation of high-density genotypes in the Fleckvieh cattle population
}

\author{
Hubert Pausch ${ }^{1 *}$, Bernhard Aigner ${ }^{1}$, Reiner Emmerling ${ }^{2}$, Christian Edel ${ }^{2}$, Kay-Uwe Götz ${ }^{2}$ and Ruedi Fries ${ }^{1}$
}

\begin{abstract}
Background: Currently, genome-wide evaluation of cattle populations is based on SNP-genotyping using 54000 SNP. Increasing the number of markers might improve genomic predictions and power of genome-wide association studies. Imputation of genotypes makes it possible to extrapolate genotypes from lower to higher density arrays based on a representative reference sample for which genotypes are obtained at higher density.

Methods: Genotypes using 639214 SNP were available for 797 bulls of the Fleckvieh cattle breed. The data set was divided into a reference and a validation population. Genotypes for all SNP except those included in the BovineSNP50 Bead chip were masked and subsequently imputed for animals of the validation population. Imputation of genotypes was performed with Beagle, findhap.f90, MaCH and Minimac. The accuracy of the imputed genotypes was assessed for four different scenarios including 50, 100, 200 and 400 animals as reference population. The reference animals were selected to account for $78.03 \%, 89.21 \%, 97.47 \%$ and $>99 \%$ of the gene pool of the genotyped population, respectively.

Results: Imputation accuracy increased as the number of animals and relatives in the reference population increased. Population-based algorithms provided highly reliable imputation of genotypes, even for scenarios with 50 and 100 reference animals only. Using MaCH and Minimac, the correlation between true and imputed genotypes was $>0.975$ with 100 reference animals only. Pre-phasing the genotypes of both the reference and validation populations not only provided highly accurate imputed genotypes but was also computationally efficient. Genome-wide analysis of imputation accuracy led to the identification of many misplaced SNP.
\end{abstract}

Conclusions: Genotyping key animals at high density and subsequent population-based genotype imputation yield high imputation accuracy. Pre-phasing the genotypes of the reference and validation populations is computationally efficient and results in high imputation accuracy, even when the reference population is small.

\section{Background}

With the availability of dense marker panels, assessing the genetic value of individuals without relying on phenotypic information is possible [1]. Current routine genomic evaluation of cattle populations is performed using the genotypes of $\sim 54000$ SNP. However, the most recent high-density genotyping arrays facilitate the highthroughput interrogation of 648874 and 777962 SNP, respectively [2]. Using densely spaced marker maps increases the probability of co-segregation of SNP and quantitative trait nucleotides (QTN) [3]. Since both genomic predictions and genome-wide association studies

\footnotetext{
* Correspondence: hubert.pausch@tierzucht.tum.de

'Lehrstuhl fuer Tierzucht, Technische Universitaet Muenchen, 85354, Freising, Germany

Full list of author information is available at the end of the article
}

exploit linkage disequilibrium (LD) between anonymous markers and QTN, increasing the density of SNP maps is likely to improve the capacities of genome-wide population analyses [4-9]. However, the relationship between validation and calibration populations is crucial to obtain accurate genomic predictions [10].

Genotype imputation is invaluable to combine different marker panels and to infer missing genotypes [11]. Imputation of genotypes makes it possible to extrapolate genotypes from lower to higher density arrays based on a representative sample of individuals genotyped at highdensity. Different approaches for imputation of genotypes exploit pedigree information [12], population-wide LD (e.g. $[13,14])$ or both sources of information (e.g. [15]).

The accuracy of genotype imputation depends on the proportion of missing genotypes [16] and the number of

\section{Biomed Central}

(c) 2013 Pausch et al.; licensee BioMed Central Ltd. This is an Open Access article distributed under the terms of the Creative Commons Attribution License (http://creativecommons.org/licenses/by/2.0), which permits unrestricted use, distribution, and reproduction in any medium, provided the original work is properly cited. 
individuals and relatives genotyped at high-density $[17,18]$. However, the number of reference genotypes required to ensure high imputation quality varies across populations and depends mainly on the effective population size [19]. Careful selection of animals for high-density genotyping facilitates population-wide imputation of high-quality genotypes while minimizing genotyping costs [20,21].

Here, we report the evaluation of four tools for imputation of genotypes in 797 Fleckvieh (FV) bulls genotyped with 639214 SNP. We show that imputation based on pre-phasing results in high accuracy and is computationally efficient. As few as 100 informative reference animals were sufficient to genotype the entire population with high accuracy.

\section{Methods}

\section{Animals}

A total of 814 bulls of the FV bovine breed were genotyped with the Illumina BovineHD Bead chip including genotypes of 777962 SNP. The animals were born between 1970 and 2007 with 90.2\% born between 1997 and 2004 (see Additional file 1). The bulls descended from 209 sires and 223 maternal grand-sires. The paternal half-sib and maternal grand-sire families comprised up to 27 and 46 members with an average of 3.9 and 3.2 members, respectively.

\section{Genotypes and quality control}

Genotype calling was performed using the default parameters of Illumina's BeadStudio. The chromosomal position of the SNP was determined based on the UMD3.1 assembly of the bovine genome [22]. We excluded 1224 Y-chromosome, 343 mitochondrial and 1735 SNP with an unknown chromosomal position from further analysis. One SNP out of 55 pairs of SNP with identical chromosomal positions but different SNP-ids (duplicates) was omitted. Eight bulls were excluded because genotyping failed for more than $5 \%$ of the SNP. We omitted 10751 SNP because genotyping failed in more than $5 \%$ of the individuals, 124 652 SNP that had a minor allele frequency (MAF) $<1 \%$ and 4024 SNP with a significant $\left(\mathrm{P}<10^{-6}\right)$ deviation from the Hardy-Weinberg equilibrium. Pedigree-based relationships among the animals were obtained using PyPedal [23], tracing pedigree information back to 1920 . Comparing the pedigree and the realized genomic relationships [24] led to the exclusion of nine animals showing major discrepancies (see Additional file 2). Genotypes of 228 sire-offspring pairs were inspected for mendelian errors (e.g. genotype $\mathrm{AA}$ and $\mathrm{BB}$ in sire and offspring, respectively). The number of mendelian errors ranged from 24 to 132 , with an average of 63 errors per pair. Genotypes of both sire and offspring were set to missing for SNP with mendelian errors. The final data set comprised
797 animals and 639214 SNP, with an average call-rate of $99.48 \%$ per individual.

\section{Evaluation of imputation accuracy}

The high-density data set was divided into a reference and a validation population. Complete genotype information was retained for animals in the reference population, whereas genotypes were set to missing for animals in the validation population for all SNP except those included in the BovineSNP50 Bead chip (version 2). SNP that are present in the BovineSNP50 Bead chip but not in the BovineHD Bead chip were not considered. Subsequently, genotype imputation was performed to infer the masked genotypes in silico. Imputation accuracy was assessed by comparing the imputed genotypes/alleles with the true genotypes/alleles and by calculating the correlation between true and imputed genotypes $\left(\mathrm{r}_{\mathrm{TG}, \mathrm{IG}}\right)$ [25]. The SNP-specific imputation accuracy was assessed as a function of allele frequencies. Allele frequencies and the corresponding proportion of correctly imputed alleles were fitted with a local regression model (LOESS), with a smoothing factor of 0.1 . The individual-specific imputation accuracy was calculated as a function of the number of relatives in the reference population. The relationship was obtained from the pedigree-based numerator relationship matrix (see above).

\section{Selection of reference animals}

Imputation accuracy was evaluated for four scenarios with an increasing number of reference animals. Animals for the reference population were selected based on $\mathbf{p}_{\mathrm{m}}=\mathbf{A}_{\mathrm{m}}^{-1} \mathbf{c}_{\mathrm{m}}$ [20], where $\mathbf{A}_{\mathrm{m}}$ is a subset of the numerator relationship matrix, $\mathbf{c}$ is a vector representing the average relationship of $\mathrm{m}$ selected animals with the entire population and $\mathbf{p}$ is a vector of the proportion of the gene pool captured by the $\mathrm{m}$ animals. Out of 797 animals with high-density genotypes, the most informative 50, 100, 200 and 400 animals were iteratively chosen to maximise $\sum_{i=1}^{m} \mathbf{p}_{i}$, i.e. the most informative 50 animals were a subset of the most informative 100 animals etc. Such subsets of animals capture the greatest proportion of the gene pool of the entire dataset and should provide the most accurate genotype imputation. Animals identified in this way were considered as reference individuals. The remaining 747, 697, 597 and 397 animals were used as validation individuals. Imputation accuracy was also assessed using randomly selected reference animals. Fifty animals were randomly selected as reference individuals and the remaining 747 animals were used as validation individuals. The random selection of reference animals and subsequent genotype imputation and validation were repeated ten times. 


\section{Imputation algorithms}

The performance of four imputation tools was evaluated. Three population-based imputation algorithms (Beagle (version 3.2.1) [13], $\mathrm{MaCH}$ (version 1.0.16.a) [14], Minimac [26]) exploiting LD were applied without considering pedigree information. Additionally, the performance of an algorithm based on long-range phasing implemented in findhap.f90 (version 2) [6], combining both family and population-based imputation, was evaluated. Beagle and $\mathrm{MaCH}$ were applied since these algorithms provide high imputation accuracy in both livestock and human populations [27,28]. Beagle and $\mathrm{MaCH}$ are imputation algorithms based on a hidden Markov model (HMM). Beagle performs a local clustering of haplotypes at each marker position to define the hidden states whereas $\mathrm{MaCH}$ samples pairs of known haplotypes for each individual based on the observed genotypes in each round of the HMM. A detailed review of the implemented algorithms is given in [29]. $\mathrm{MaCH}$ is time consuming, especially for large reference populations [30]. Thus, we also evaluated Minimac, a computationally efficient "prephasing"-based implementation of the $\mathrm{MaCH}$ algorithm, taking haplotypes as input for both the reference and the validation populations. Therefore, haplotypes for the reference and validation populations were inferred using Beagle and subsequent haplotype-based imputation of missing genotypes was performed with Minimac. While Beagle, $\mathrm{MaCH}$ and Minimac provide allele dosage data (i.e. continuously distributed values ranging from 0 to 2 ), findhap. $f 90$ provides discrete genotypes only (i.e. 0,1,2). For the present study, discrete genotypes were analysed and missing alleles resulting from imputation with findhap. $f 90$ were subsequently imputed based on allele frequencies. All programs were run on an Intel Xeon 2.13 Ghz processor using recommended parameters. A detailed overview of the parameters applied with the different tools is given in Additional file 3.

\section{Identification of misplaced SNP}

Genome-wide analysis of imputation accuracy detected regions of poor imputation quality, most likely because of misplacement of SNP. The chromosomes were partitioned into segments of $0.5 \mathrm{Mb}$. The segments were inspected for SNP with more than 10\% incorrectly imputed genotypes. If more than ten SNP with more than $10 \%$ of incorrectly imputed genotypes were located within a segment, all the SNP within this segment were considered as misplaced. If less than ten SNP per segment had more than $10 \%$ incorrectly imputed genotypes, only the erroneously imputed SNP were considered as misplaced.

\section{Results}

Genotypes for animals of the validation population were imputed based on an increasing number of highly informative reference animals with high-density genotypes. $78.03 \%$ of the genes/haplotypes of the 797 studied animals could be traced back to the subset of the 50 most informative reference animals. This fraction increased to $89.21 \%, 97.47 \%$ and $>99 \%$ for the scenarios including 100, 200 and 400 reference animals. Most of the 797 animals (90.2\%) were born between 1997 and 2004 (see Additional file 1) and the number of sireoffspring pairs was low $(n=228)$. Within the subset of the 50 most informative animals, the majority (56\%) was born before 1997. These animals can be considered as the ancestors of the studied population. Most validation animals had no first-degree relatives in the reference population (Table 1). The fraction of validation animals without relatives with a pedigree relationship greater than 0.25 was $18.07 \%$ (135 of 747 ) and $3.78 \%$ (15 of 397) in the scenarios including 50 and 400 reference animals, respectively. However, the average number of related reference animals was very small for the validation animals across all scenarios (Table 2). Imputation of genotypes was performed separately for six chromosomes (Table 3). The number of masked and subsequently imputed high-density genotypes ranged from 93.44\% (BTA1) to $94.41 \%$ (BTA5). The validation populations contained no missing genotypes after imputation with Beagle, MaCH and Minimac. However, 6.49\%, 1.46\%, $0.26 \%$ and $0.11 \%$ of the masked genotypes remained missing after imputation with findhap. 990 for the scenarios including 50, 100, 200 and 400 reference animals, respectively. Those genotypes were subsequently imputed based on allele frequencies.

\section{Genotypic imputation accuracy}

The imputation accuracy increased as the size of the reference population increased (Figure 1). However, the correlation between true and imputed genotypes $\left(\mathrm{r}_{\mathrm{TG}, \mathrm{IG}}\right)$ varied considerably across chromosomes, especially for the scenario including 50 reference animals. With Beagle, the correlation ranged from 0.825 (BTA25) to 0.896 (BTA1) and with findhap.f90 it ranged from 0.793 (BTA25) to 0.899 (BTA2). $\mathrm{MaCH}$ and Minimac provided nearly identical imputation quality, although computational time was considerably lower with Minimac (Table 4). Phasing both the reference and validation populations with Beagle and subsequent haplotypebased genotype imputation with Minimac outperformed all other approaches, especially when the number of reference animals was small. Imputation with Minimac yielded an $\mathrm{r}_{\mathrm{TG}, \mathrm{IG}}$ value of 0.953 with 50 reference animals, while with $\mathrm{MaCH}$, Beagle and findhap.f90 $\mathrm{r}_{\mathrm{TG}, \mathrm{IG}}$ was only $0.945,0.858$ and 0.865 , respectively (Table 5). Increasing the number of reference animals to 100, 200 and 400 yielded an $r_{\mathrm{TG}, \mathrm{IG}}$ value of $0.977,0.989$ and 0.993 with Minimac. 
Table 1 Number of validation animals without close relatives in the reference population

\begin{tabular}{|c|c|c|c|c|}
\hline & \multicolumn{4}{|c|}{ Scenario (number of reference animals / number of validation animals) } \\
\hline & $50 / 747$ & $100 / 697$ & $200 / 597$ & $400 / 397$ \\
\hline no relatives with $r \geq 0.50$ & 621 & 562 & 453 & 316 \\
\hline no relatives with $r \geq 0.25$ & 135 & 62 & 30 & 15 \\
\hline no relatives with $r \geq 0.125$ & 16 & 4 & - & - \\
\hline no relatives with $r \geq 0.0625$ & 5 & 2 & - & - \\
\hline
\end{tabular}

The number of validation animals without close relatives in the reference population is presented for four different classes of relationship ( $r$ ) and four scenarios with an increasing number of reference animals. Since most animals in our study were born between 1997 and 2004 , the number of validation animals without close relatives in the reference population was very high across all scenarios.

The approach based on pre-phasing implemented in Minimac not only provided highly accurate imputed genotypes but was also computationally efficient (Table 4). However, findhap. $f 90$ was the most efficient computationally, especially for a large number of reference genotypes.

\section{Allelic imputation accuracy}

The proportion of correctly imputed alleles was $~ 98.5 \%$ for $\mathrm{MaCH}$ and Minimac, for the scenario with 100 reference animals (Table 5). The corresponding $\mathrm{r}_{\mathrm{TG}, \mathrm{IG}}$ was $>0.975$. $\mathrm{MaCH}$ and Minimac clearly outperformed Beagle and findhap. $f 90$ in all scenarios and provided the most accurate results for rare alleles. Imputation quality for rare alleles was very poor with findhap. $f 90$ (Figure 2). All algorithms inferred frequent alleles with high quality even when the number of reference animals was small.

\section{Individual imputation accuracy}

The quality of the imputed genotypes varied considerably between animals (Figure 3A). The extent of genotype information from relatives in the reference population was the major determinant of the individual imputation accuracy. For the scenario with 50 reference animals, most validation animals $(n=621)$ had no firstdegree relatives in the reference population (Table 1). We considered that reference and validation animals are close relatives if their relationship was above 0.12 . While $\mathrm{r}_{\mathrm{TG}, \mathrm{IG}}$ was $<0.90$ for all algorithms without including high-density genotype information from closely related animals, the imputation accuracy increased considerably as the number of relatives in the reference population increased (Figure 3B). $\mathrm{MaCH}$ and Minimac provided highly accurate genotypes, even if the number of relatives in the reference population was small. With $\mathrm{MaCH}$ and Minimac, the average $\mathrm{r}_{\mathrm{TG}, \mathrm{IG}}$ exceeded 0.94 for validation animals which had at least one related animal with $r \geq 0.12$ in the reference population. The accuracy of findhap. $f 90$ increased considerably as the number of relatives in the reference population increased. With Beagle, $\mathrm{r}_{\mathrm{TG}, \mathrm{IG}}$ never exceeded 0.885 for the scenario with 50 reference animals.

The impact of genotype information from relatives decreased as the size of the reference population increased when applying the population-based imputation tools Beagle, $\mathrm{MaCH}$ and Minimac. In contrast, the quality of the imputed genotypes obtained with findhap. $f 90$ increased considerably as the number of relatives increased, even for the scenario with 400 reference animals (see Additional file 4).

\section{Imputation accuracy obtained based on randomly selected reference animals}

Ten subsets of 50 randomly selected animals that explained between $68.9 \%$ and $71.9 \%$ of the gene pool of the entire data set were used as reference populations to impute genotypes for 747 validation animals on chromosome 20. The imputation accuracy obtained based on these randomly selected reference animals was lower compared with $\mathrm{r}_{\mathrm{TG}, \mathrm{IG}}$ obtained using the most informative animals as reference individuals across all replications for the four imputation tools (Table 6). Using the most informative instead of random animals as reference population increased $\mathrm{r}_{\mathrm{TG}, \mathrm{IG}}$ especially for findhap.f 90

Table 2 Average number of relatives in the reference population

\begin{tabular}{lcccc}
\hline $\begin{array}{l}\text { Scenario } \\
\text { (reference animals / validation animals) }\end{array}$ & \multicolumn{3}{c}{ Average number of relatives in the reference population } \\
\cline { 2 - 5 } & $\mathbf{r} \geq \mathbf{0 . 5 0}$ & $\mathbf{0 . 2 5} \geq \mathbf{r}<\mathbf{0 . 5 0}$ & $\mathbf{0 . 1 2 5} \geq \mathbf{r}<\mathbf{0 . 2 5}$ & $\mathbf{0 . 0 6 2 5} \geq \mathbf{r}<\mathbf{0 . 1 2 5}$ \\
\hline $50 / 747$ & 0.18 & 1.15 & 1.86 & 4.57 \\
$100 / 697$ & 0.21 & 1.61 & 2.43 & 8.64 \\
$200 / 597$ & 0.27 & 2.51 & 4.16 & 20.89 \\
$400 / 397$ & 0.27 & 4.82 & 9.60 & 54.58 \\
\hline
\end{tabular}

The average number of relatives in the reference population is given for the animals in the validation population for four classes of relationships ( $r$ ) and four scenarios with an increasing number of reference animals. The average number of close relatives in the reference population was very small for most animals in the validation population. 
Table 3 Number of SNP used for the evaluation of imputation accuracy on six chromosomes

\begin{tabular}{cccccc}
\hline Chr & $\begin{array}{c}\text { Chromosome-length } \\
\text { [Mb] }\end{array}$ & $\begin{array}{c}\text { Number of high-density } \\
\text { SNP in the reference } \\
\text { population }\end{array}$ & $\begin{array}{c}\text { Average distance } \\
\text { between two high-density } \\
\text { SNP [bp] }\end{array}$ & $\begin{array}{c}\text { Number of medium-density } \\
\text { SNP in the validation } \\
\text { population }\end{array}$ & $\begin{array}{c}\text { Average distance between } \\
\text { two medium-density } \\
\text { SNP [bp] }\end{array}$ \\
\hline 1 & 158.32 & 39167 & 4042 & 2568 & 61587 \\
5 & 121.18 & 29050 & 4171 & 1621 & 74740 \\
10 & 104.30 & 26695 & 3906 & 1646 & 62724 \\
15 & 85.27 & 21425 & 3978 & 1280 & 65850 \\
20 & 71.98 & 19111 & 3764 & 1183 & 60530 \\
25 & 42.85 & 11725 & 3648 & 744 & 57533
\end{tabular}

Number of high-density SNP passing stringent quality parameters for the six evaluated chromosomes. The medium-density SNP are a subset of the bovineHD BeadChip collection that are interrogated with the BovineSNP50 BeadChip (version 2). SNP positions were determined based on the UMD3.1 assembly of the bovine genome.

(0.876 vs. 0.837). However, Minimac and MaCH provided high imputation accuracy even with randomly selected reference populations.

\section{Identification of misplaced SNP}

Minimac was used for genome-wide imputation of highdensity genotypes. Of 639214 SNP, 39679 SNP were retained for 397 validation animals, while genotypes for the remaining 599535 SNP were imputed using 400 reference animals. With this design $\mathrm{r}_{\mathrm{TG}, \mathrm{IG}}$ was equal to 0.993 across six analysed chromosomes (Table 5). The genome-wide distribution of the imputation accuracy revealed genomic regions with poor imputation quality (Figure 4). Analysis of these regions showed that misplacement of SNP increased the fraction of poorly imputed genotypes. Poor imputation quality along entire chromosomal segments results from intra-chromosomal

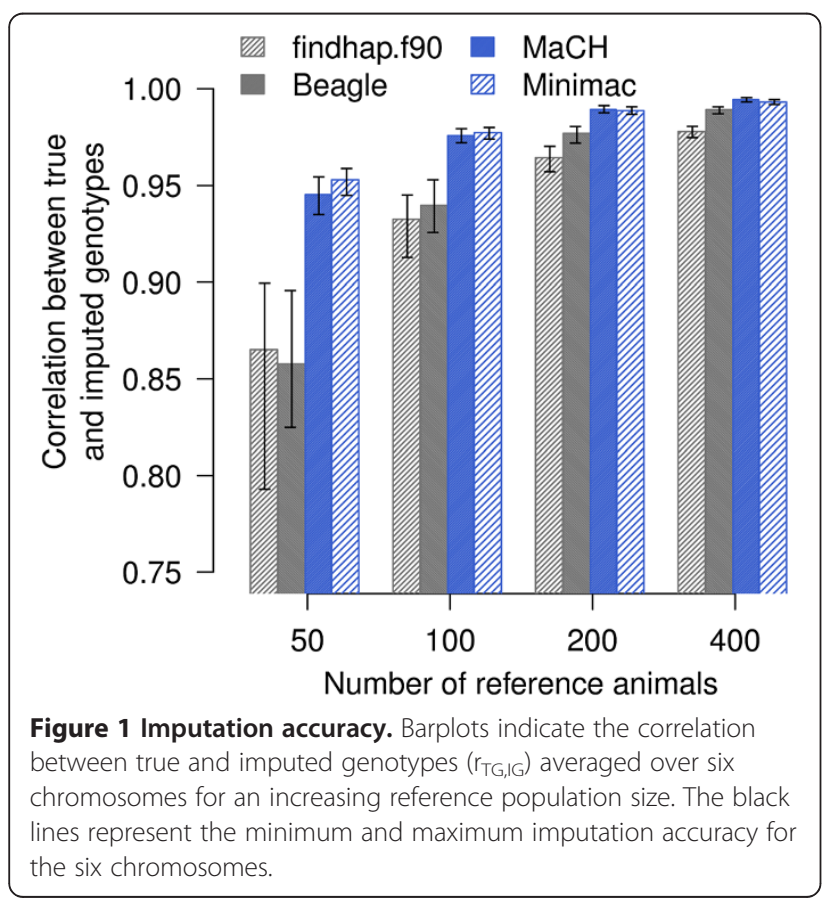

misplacement of adjacent SNP. However, analysis of LD also indicated inter-chromosomal misplacement of individual SNP (see Additional file 5). A total of 5039 out of 599535 SNP (0.84\%) was identified as probably misplaced using this procedure.

\section{Discussion}

Four imputation tools were evaluated using a data set consisting of 797 bulls of the German FV population genotyped at 639214 SNP. The reference animals were selected to capture the greatest proportion of the gene pool of the genotyped population. Using Minimac, up to 97.1\% of the alleles were correctly imputed based on 50 pre-selected reference animals. Imputation accuracy based on genotypes of randomly selected reference animals was slightly, albeit consistently lower (Table 6). Brøndum et al. [31] used Beagle to impute high-density genotypes in three cattle breeds based on $\sim 200$ reference animals and obtained $\mathrm{r}_{\mathrm{TG}, \mathrm{IG}}$ ranging from 0.925 to 0.973 . In our study, Beagle yielded an $\mathrm{r}_{\mathrm{TG}, \mathrm{IG}}$ of 0.977 with 200 pre-selected reference animals. In the Holstein-Friesian breed, Erbe et al. [32] obtained $97.7 \%$ of correctly imputed genotypes with Beagle using 400 randomly selected reference animals. In our study, using Beagle with 400 pre-selected animals as reference population yielded $98.7 \%$ of correctly imputed genotypes. However, $\mathrm{MaCH}$ and Minimac yielded the same imputation accuracy with 200 reference animals only. Selecting highly informative reference animals (i.e. key animals) maximises the proportion of genes/haplotypes in the validation population that can be traced back to these key animals and thus maximises imputation accuracy while minimizing genotyping costs [20,33,34]. Our findings demonstrate that pre-selecting highly-informative reference animals is slightly beneficial for subsequent genotype imputation. The most influential animals have been identified in various cattle populations $[35,36]$ and such 'key animals' will be used for whole-genome re-sequencing. Simulations have shown that imputation of sequence information from a restricted number of highly informative 
Table 4 Computing time for the imputation of high-density SNP on chromosomes 1, 15 and 25

\begin{tabular}{|c|c|c|c|c|c|}
\hline $\begin{array}{c}\text { Number of animals in reference } \\
\text { and validation population }\end{array}$ & Chr & Beagle & $\mathrm{MaCH}^{\mathrm{a}}$ & findhap. $f 90^{\mathrm{b}}$ & Minimac $^{c}$ \\
\hline \multirow[t]{3}{*}{$50 / 747$} & BTA1 & $2.67 \mathrm{~h}$ & 1.30 h (0.03 h / 0.30 h / 0.97 h) & $0.07 \mathrm{~h}$ & 0.17 h (0.03 h / 0.07 h / 0.07 h) \\
\hline & BTA15 & $1.18 \mathrm{~h}$ & 0.68 h (0.02 h / 0.14 h / 0.52 h) & $0.04 \mathrm{~h}$ & 0.09 h (0.02 h / 0.04 h / 0.03 h) \\
\hline & BTA25 & $0.67 \mathrm{~h}$ & 0.37 h (0.01 h / 0.08 h / 0.28 h) & $0.04 \mathrm{~h}$ & 0.05 h (0.01 h / 0.02 h / 0.02 h) \\
\hline \multirow[t]{3}{*}{$100 / 697$} & BTA1 & $3.93 \mathrm{~h}$ & 5.01 h (0.08 h / 1.11 h / 3.82 h) & $0.07 \mathrm{~h}$ & 0.27 h (0.08 h / 0.06 h / 0.13 h) \\
\hline & BTA15 & $2.48 \mathrm{~h}$ & 2.72 h (0.05 h / 0.55 h / 2.12 h) & $0.05 \mathrm{~h}$ & 0.15 h (0.05 h / 0.03 h / 0.07 h) \\
\hline & BTA25 & $1.33 \mathrm{~h}$ & 1.48 h (0.03 h / 0.32 h / 1.13 h) & $0.04 \mathrm{~h}$ & 0.09 h (0.03 h / 0.02 h / 0.04 h) \\
\hline \multirow[t]{3}{*}{$200 / 597$} & BTA1 & $4.49 \mathrm{~h}$ & $18.92 \mathrm{~h}(0.20 \mathrm{~h} / 4.31 \mathrm{~h} / 14.41$ h) & $0.07 \mathrm{~h}$ & 0.48 h (0.20 h / 0.05 h / 0.23 h) \\
\hline & BTA15 & $2.87 \mathrm{~h}$ & 10.06 h (0.11 h / 2.22 h / 7.73 h) & $0.05 \mathrm{~h}$ & $0.27 \mathrm{~h}(0.11 \mathrm{~h} / 0.03 \mathrm{~h} / 0.13 \mathrm{~h})$ \\
\hline & BTA25 & $1.38 \mathrm{~h}$ & $5.76 \mathrm{~h}(0.06 \mathrm{~h} / 1.24 \mathrm{~h} / 4.45 \mathrm{~h})$ & $0.04 \mathrm{~h}$ & $0.14 \mathrm{~h}(0.06 \mathrm{~h} / 0.01 \mathrm{~h} / 0.07 \mathrm{~h})$ \\
\hline \multirow[t]{3}{*}{400 / 397} & BTA1 & $3.73 \mathrm{~h}$ & 81.23 h (0.44 h / 21.97 h / 58.82 h) & $0.07 \mathrm{~h}$ & $1.1 \mathrm{~h}(0.44 \mathrm{~h} / 0.03 \mathrm{~h} / 0.63 \mathrm{~h})$ \\
\hline & BTA15 & $2.45 \mathrm{~h}$ & 40.16 h (0.21 h / 10.52 h / 29.43 h) & $0.05 \mathrm{~h}$ & $0.56 \mathrm{~h}(0.21 \mathrm{~h} / 0.02 \mathrm{~h} / 0.33 \mathrm{~h})$ \\
\hline & BTA25 & $1.37 \mathrm{~h}$ & 28.30 h (0.11 h / 5.98 h / 22.21 h) & $0.04 \mathrm{~h}$ & $0.30 \mathrm{~h}(0.11 \mathrm{~h} / 0.01 \mathrm{~h} / 0.18 \mathrm{~h})$ \\
\hline
\end{tabular}

The number of imputed SNP was 36 599, 20145 and 10981 for chromosomes 1, 15 and 25, respectively. Computing was performed on an Intel Xeon 2.13 Ghz processor.

${ }^{a}$ The entire computing time for $\mathrm{MaCH}$ can be partitioned into three separate steps (in parentheses): pre-phasing of the reference population with Beagle, inference of tuning parameters based on 200 randomly selected animals of the validation population and actual genotype imputation with $\mathrm{MaCH}$.

b findhap.f90 was run exploiting the multi-threading option.

' The entire computing time for Minimac can be partitioned into three separate steps (in parentheses): pre-phasing of the reference population with Beagle, prephasing of the validation population with Beagle and actual genotype imputation with Minimac.

individuals is feasible [21]. Genotyping a large number of animals at high-density and subsequently imputing the whole-genome sequence information from a small number of carefully selected 'key animals' might lead to even higher accuracy, since imputation quality strongly depends on the marker density in both reference and validation populations $[34,37,38]$. However, our findings also show that the choice of a suitable imputation algorithm is more crucial than the selection of 'key animals' to obtain high imputation accuracy based on a small number of reference animals.

Imputation accuracy increased as the size of the reference population increased, which agrees with $[17,37,39]$. However, the performance of imputation tools varied considerably, especially when the number of animals and relatives with high-density genotypes was limited. $\mathrm{MaCH}$ and Minimac provided highly accurate imputed genotypes, even with only 50 reference animals compared to Beagle and findhap.f90. This advantage of $\mathrm{MaCH}$ and
Minimac for genotype imputation based on a small reference population agrees with the reports of Browning and Browning [40] and Pei et al. [27]. If the size of the reference population increases, the accuracies of the imputation tools converge, which agrees with findings of Browning and Browning [13]. For the scenarios with 50 and 100 reference animals, the approach based on prephasing and implemented with Minimac provided the most accurate genotypes. Minimac was run after phasing both reference and validation populations with Beagle, disregarding pedigree information. Accounting for pedigree information might further improve the quality of phasing and thus the accuracy of subsequent genotype imputation $[15,16,30]$. In our study, the number of validation animals with close relatives in the reference population was very small. Thus, we found no increase in imputation accuracy with findhap.f90. However, if the number of closely related reference animals is increased, imputation algorithms using both pedigree and population

Table 5 Evaluation of imputation accuracy

\begin{tabular}{|c|c|c|c|c|c|c|c|c|c|c|c|c|}
\hline \multirow{2}{*}{$\begin{array}{l}\text { Number of } \\
\text { animals in reference } \\
\text { and validation population }\end{array}$} & \multicolumn{3}{|c|}{ Beagle } & \multicolumn{3}{|c|}{$\mathrm{MaCH}$} & \multicolumn{3}{|c|}{ findhap.f90 } & \multicolumn{3}{|c|}{ Minimac } \\
\hline & $\begin{array}{l}\text { Correct } \\
\text { alleles }\end{array}$ & $\begin{array}{c}\text { Correct } \\
\text { genotypes }\end{array}$ & $r_{\mathrm{TG}, \mathrm{IG}}$ & $\begin{array}{l}\text { Correct } \\
\text { alleles }\end{array}$ & $\begin{array}{c}\text { Correct } \\
\text { genotypes }\end{array}$ & $r_{T G, I G}$ & $\begin{array}{l}\text { Correct } \\
\text { alleles }\end{array}$ & $\begin{array}{l}\text { Correct } \\
\text { genotypes }\end{array}$ & $r_{\mathrm{TG}, \mathrm{IG}}$ & $\begin{array}{l}\text { Correct } \\
\text { alleles }\end{array}$ & $\begin{array}{c}\text { Correct } \\
\text { genotypes }\end{array}$ & $\mathrm{r}_{\mathrm{TG}, \mathrm{IG}}$ \\
\hline $50 / 747$ & 0.914 & 0.840 & 0.858 & 0.966 & 0.933 & 0.945 & 0.925 & 0.858 & 0.865 & 0.971 & 0.942 & 0.953 \\
\hline 100 / 697 & 0.963 & 0.927 & 0.940 & 0.985 & 0.970 & 0.976 & 0.959 & 0.921 & 0.933 & 0.986 & 0.972 & 0.977 \\
\hline $200 / 597$ & 0.986 & 0.972 & 0.977 & 0.993 & 0.987 & 0.989 & 0.978 & 0.956 & 0.965 & 0.993 & 0.986 & 0.989 \\
\hline $400 / 397$ & 0.993 & 0.987 & 0.989 & 0.996 & 0.993 & 0.994 & 0.986 & 0.973 & 0.978 & 0.996 & 0.992 & 0.993 \\
\hline
\end{tabular}

The mean allelic and genotypic accuracies over six chromosomes (BTA1, BTA5, BTA10, BTA15, BTA20, BTA25) were assessed for the imputed genotypes based on an increasing size of the reference population. Additionally, the correlation between true and imputed genotypes $\left(r_{\mathrm{TG}, \mathrm{IG}}\right)$ was calculated.

a genotype is correctly imputed if both alleles are correctly imputed. 


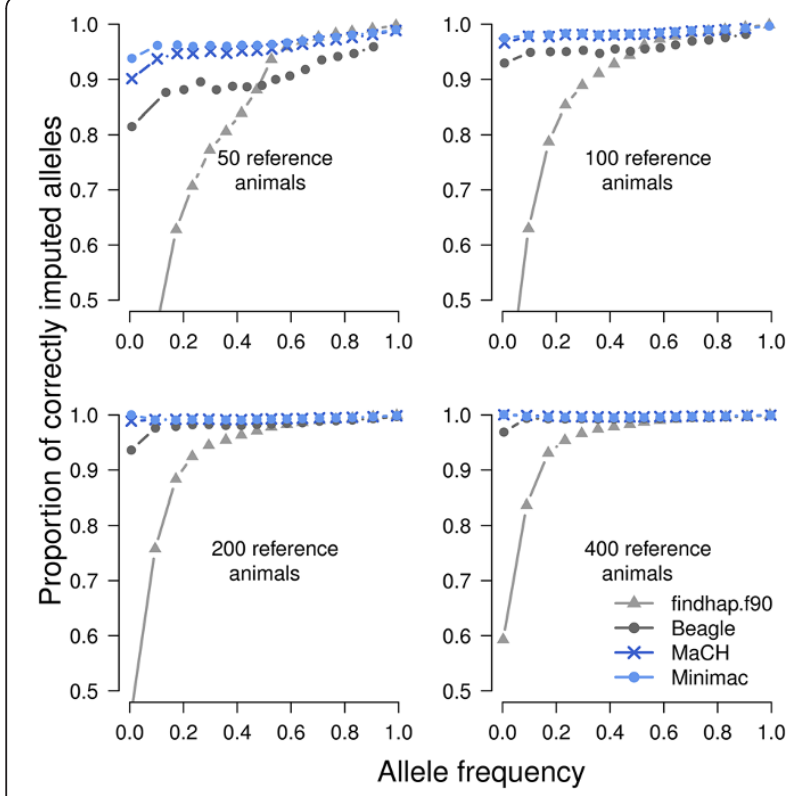

Figure 2 Allelic imputation accuracy. The proportion of correctly imputed alleles is displayed as a function of allele frequencies for findhap.f90 (light grey), Beagle (dark grey), MaCH (blue) and Minimac (light blue) for an increasing reference population size. The curves were obtained by fitting a nonparametric local regression (LOESS).

information are likely to outperform tools using population information only [41]. The pre-phasing approach applied in the present study is preferable when the number of related reference animals is small. Besides allowing for a high imputation accuracy, imputation approaches based on prephasing are computationally efficient. The reference genotypes need to be phased only once and the phasing step can be separated from the actual imputation step [26,42]. This restricts the computational burden of genotype imputation in routine implementations such as genomic prediction. Previous studies have shown that long-range phasing and haplotype library imputation provide accurately imputed
Table 6 Imputation accuracy on chromosome 20 based on varying reference populations

\begin{tabular}{lcccc}
\hline & $\begin{array}{c}\mathbf{5 0} \text { most } \\
\text { informative } \\
\text { animals }\end{array}$ & \multicolumn{3}{c}{$\mathbf{5 0}$ randomly selected animals } \\
\cline { 3 - 5 } & 0.866 & Mean & Min & Max \\
\hline Beagle & 0.949 & 0.942 & 0.841 & 0.864 \\
MaCH & 0.876 & 0.837 & 0.812 & 0.937 \\
findhap.f90 & 0.957 & 0.947 & 0.943 & 0.951 \\
Minimac & &
\end{tabular}

The correlation between true and imputed genotypes $\left(\mathrm{r}_{\mathrm{TG}, \mathrm{GG}}\right)$ based on the 50 most informative animals as reference population is compared with $\mathrm{r}_{\mathrm{TG}, \mathrm{IG}}$ obtained with 50 randomly selected reference animals. The mean, minimum and maximum $r_{\mathrm{TG}, \mathrm{G}}$ obtained with randomly selected reference animals are displayed across ten replications for the four imputation tools.

genotypes in livestock populations at a low computational input when the reference population is large [6,43-45]. Our results indicate that pre-phasing might slightly increase imputation accuracy, particularly when the number of reference genotypes is limited. The benefit of pre-phasing is expected to result from capturing LD effects at a better resolution [26]. Thus, pre-phasing based approaches might become the method of choice to impute the entire sequence information based on the re-sequencing of a limited number of key genomes in livestock populations.

Two population-based approaches that exploit LD without explicitly considering pedigree information $(\mathrm{MaCH}$, Minimac) outperformed findhap. $f 90$ that takes relationships into account. findhap. $f 90$ was specifically designed to impute genotypes using large data sets and exploiting comprehensive pedigree information [6]. In contrast, our data set comprised 797 animals only, mainly born between 1997 and 2004. Furthermore, the number of genotyped relatives in the reference population was very small for most of the animals in the validation population, resulting in comparably low overall imputation accuracy when using findhap. $f 90$. However, imputation with findhap. $f 90$ provided $>98 \%$ of correctly
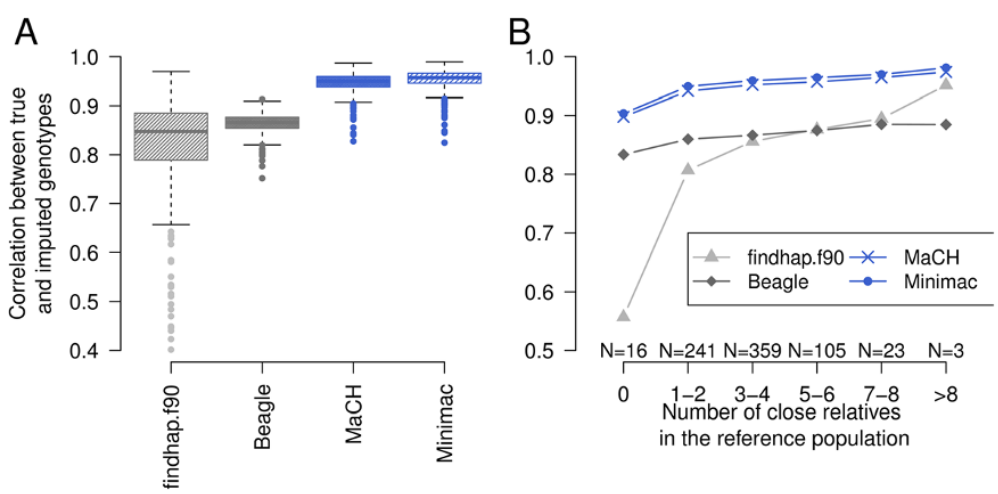

Figure $\mathbf{3}$ Individual imputation accuracy for the scenario with $\mathbf{5 0}$ reference animals. Barplots indicate the correlation between true and imputed genotypes $\left(r_{\mathrm{TG}, \mathrm{IG}}\right)$ for 747 animals based on 50 reference animals $(\mathbf{A})$. The individual $\mathrm{r}_{\mathrm{TG}, \mathrm{IG}}$ increased considerably as the number of close relatives increased (coefficient of relationship $>0.12$ ) in the reference population $(\mathbf{B})$. 


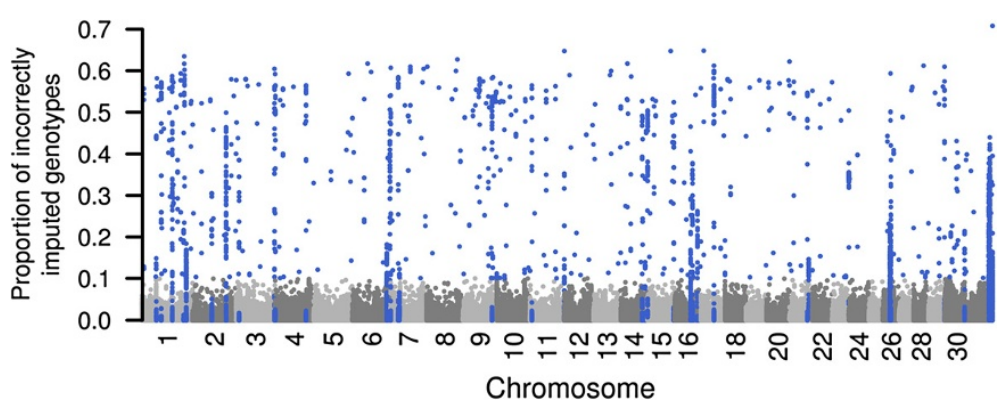

Figure 4 Genome-wide distribution of the proportion of correctly imputed genotypes. Genotypes of 599535 SNP were imputed for 397 animals based on haplotype information of 400 reference animals using Minimac. Blue dots represent 5039 SNP within regions of poor imputation quality probably representing misplaced SNP.

imputed genotypes when a substantial number of the relatives with genotypes was present in the reference population (see Additional file 4). This agrees with findings for the American Holstein-Friesian population [6]. However, comparing imputation accuracy across studies and breeds is difficult since data sets and populationspecific parameters (e.g. LD, effective population size $\left(\mathrm{N}_{\mathrm{e}}\right)$ ) might differ substantially. While recent $\mathrm{N}_{\mathrm{e}}$ estimates for the Holstein-Friesian population range from $<100$ to 114 [35,46,47], $\mathrm{N}_{\mathrm{e}}$ for the FV population is considerably higher (see Additional file 6). Low LD, which is typical for populations with large $\mathrm{N}_{\mathrm{e}}$ [48], complicates genotype imputation considerably [27]. However, in populations with small $\mathrm{N}_{\mathrm{e}}$ (e.g. Jersey cattle [35]), genotype imputation based on a small number of carefully selected reference animals yields a reasonable accuracy [32]. Our results demonstrate that genotyping at least 100 pre-selected animals at high density and subsequently applying population-based imputation yielded highly reliable genotypes for the analysed subset of the FV population, although $\mathrm{N}_{\mathrm{e}}$ is comparatively large. However, the animals in our study are highly selected artificial insemination bulls and might not fully reflect the haplotype diversity of the entire FV population.

Genome-wide analysis of imputation accuracy also allowed misplaced SNP to be identified. Although misplaced SNP are particularly obstructive for haplotypebased analyses (e.g. identification of selective sweeps) [49], the position of significantly associated SNP in genome-wide association studies should also be validated to avoid misinterpretations. Analysis of genomic regions with poor imputation quality revealed 5039 SNP that are most likely misplaced. Recently, Erbe et al. [32] showed similar results. The total number of misplaced SNP might be even higher, since SNP with very low MAF $(<1 \%)$ were excluded for the evaluation of imputation accuracy. Furthermore, our procedure is not suitable to reveal misplaced SNP within short distances. However, the proportion of misplaced SNP in the highdensity array used here is slightly higher than in the
BovineSNP50 Bead chip [50], which is most likely due to a better resolution of the high-density marker map. The number of misplaced SNP detected here is considerably higher than reported by Fadista and Bendixen [51], who relied on a more precise assembly of the reference sequence. However, LD-based procedures make it possible to realign SNP positions despite imperfectly assembled reference genomes.

\section{Conclusions}

Genotype imputation allows different marker panels to be combined and missing genotypes to be infered in silico. The quality of the imputed genotypes strongly depends on the amount of genotype information that is available from relatives. However, population-based imputation tools provide highly-reliable genotypes even if the number of reference animals is small. In addition, imputation accuracy increases if the animals of the reference panel are chosen to maximally contribute to the gene pool of the imputation population. Pre-phasing the genotypes of both the reference and validation populations not only results in highly accurately imputed genotypes but is also computationally efficient.

\section{Additional files}

Additional file 1: Birth years of 814 genotyped bulls of the Fleckvieh breed. Birth years ranged from 1970 to 2007 with 90.2\% of the animals born between 1997 and 2004.

Additional file 2: Pairwise pedigree vs. genomic relationship. Pairwise pedigree vs. genomic relationship for 806 Fleckvieh bulls passing stringent quality before (A) and after (B) the exclusion of nine animals with inconsistencies.

Additional file 3: Imputation pipelines for the four different imputation tools.

Additional file 4: Individual imputation accuracy for the scenario with $\mathbf{4 0 0}$ reference animals. The individual imputation accuracy $\left(r_{T G, G)}\right)$ increased only slightly with an increasing number of second-degree relatives in the reference population for Beagle, MaCH and Minimac. However, a strong increase in accuracy was observed for findhap.f90.

Additional file 5: Identification of misplaced SNP on chromosome 26. The distribution of the proportion of imputation errors highlights the 
regions with poor imputation quality on chromosome 26 (A). Blue and red symbols indicate 391 SNP that were considered as misplaced. The red symbol indicates BovineHD2600003844, which is located on BTA26 (according to the UMD3 assembly). However, analysis of linkage disequilibrium with all other SNP indicates that the proximal region of BTA11 is the actual position (B). The pairwise linkage disequilibrium on BTA26 is shown as a function of the pairwise distance before (C) and after (D) the exclusion of 391 probably misplaced SNP $\left(r^{2}\right.$-values below 0.1 are omitted)

Additional file 6: Estimation of the effective population size for the Fleckvieh population.

\section{Abbreviations}

FV: Fleckvieh; HMM: Hidden Markov model; LD: Linkage disequilibrium; MAF: Minor allele frequency; $\mathrm{N}_{\mathrm{e}}$ : Effective population size; QTN: Quantitative trait nucleotide; SNP: Single nucleotide polymorphism.

\section{Competing interests}

The authors declare that they have no competing interests.

\section{Authors' contributions}

HP and RF conceived and designed the experiments, HP and BA performed the experiments, RE, CE and KUG contributed pedigree and genotype data and HP and RF wrote the manuscript. All authors have read and approved the final manuscript.

\section{Acknowledgements}

The authors thank the associate editor and two anonymous referees for their valuable comments and suggestions. The authors acknowledge funding from the German Federal Ministry of Education and Research (BMBF) within the AgroClustEr "Synbreed - Synergistic plant and animal breeding" (grant id 0315527B)

\section{Author details}

${ }^{1}$ Lehrstuhl fuer Tierzucht, Technische Universitaet Muenchen, 85354, Freising, Germany. ${ }^{2}$ Institut fuer Tierzucht, Bayerische Landesanstalt für Landwirtschaft, 85586, Poing, Germany.

Received: 18 June 2012 Accepted: 25 January 2013

Published: 13 February 2013

\section{References}

1. Meuwissen THE, Hayes BJ, Goddard ME: Prediction of total genetic value using genome-wide dense marker maps. Genetics 2001, 157:1819-1829.

2. Rincon G, Weber KL, Van Eenennaam AL, Golden BL, Medrano JF: Hot topic: Performance of bovine high-density genotyping platforms in Holsteins and Jerseys. J Dairy Sci 2011, 94:6116-6121.

3. Khatkar MS, Nicholas FW, Collins AR, Zenger KR, Cavanagh JA, Barris W, Schnabel RD, Taylor JF, Raadsma HW: Extent of genome-wide linkage disequilibrium in Australian Holstein-Friesian cattle based on a highdensity SNP panel. BMC Genomics 2008, 9:187.

4. Spencer CCA, Su Z, Donnelly P, Marchini J: Designing genome-wide association studies: sample size, power, imputation, and the choice of genotyping chip. PLoS Genet 2009, 5:e1000477.

5. Meuwissen THE, Goddard ME: Accurate prediction of genetic values for complex traits by whole-genome resequencing. Genetics 2010, 185:623-631.

6. VanRaden PM, O'Connell JR, Wiggans GR, Weigel KA: Genomic evaluations with many more genotypes. Genet Sel Evol 2011, 43:10.

7. Su G, Brøndum RF, Ma P, Guldbrandtsen B, Aamand GP, Lund MS: Comparison of genomic predictions using medium-density $(\sim 54,000)$ and high-density $(\sim 777,000)$ single nucleotide polymorphism marker panels in Nordic Holstein and Red dairy cattle populations. J Dairy Sci 2012, 95:4657-4665.

8. Pryce JE, Arias J, Bowman PJ, Davis SR, Macdonald KA, Waghorn GC, Wales WJ, Williams YJ, Spelman RJ, Hayes BJ: Accuracy of genomic predictions of residual feed intake and 250-day body weight in growing heifers using 625,000 single nucleotide polymorphism markers. J Dairy Sci 2012, 95:2108-2119.
9. Pausch H, Wang X, Jung S, Krogmeier D, Edel C, Emmerling R, Götz K-U, Fries R: Identification of QTL for UV-protective eye area pigmentation in cattle by progeny phenotyping and genome-wide association analysis. PLoS One 2012, 7:e36346

10. Habier D, Tetens J, Seefried F-R, Lichtner P, Thaller G: The impact of genetic relationship information on genomic breeding values in German Holstein cattle. Genet Sel Evol 2010, 42:5.

11. Li Y, Willer C, Sanna S, Abecasis G: Genotype imputation. Annu Rev Genomics Hum Genet 2009, 10:387-406.

12. Abecasis GR, Cherny SS, Cookson WO, Cardon LR: Merlin-rapid analysis of dense genetic maps using sparse gene flow trees. Nat Genet 2002, 30:97-101.

13. Browning BL, Browning SR: A unified approach to genotype imputation and haplotype-phase inference for large data sets of trios and unrelated individuals. Am J Hum Genet 2009, 84:210-223.

14. Li Y, Willer CJ, Ding J, Scheet P, Abecasis GR: MaCH: using sequence and genotype data to estimate haplotypes and unobserved genotypes. Genet Epidemiol 2010, 34:816-834.

15. Druet $T$, Georges M: A hidden markov model combining linkage and linkage disequilibrium information for haplotype reconstruction and quantitative trait locus fine mapping. Genetics 2010, 184:789-798.

16. Mulder HA, Calus MPL, Druet T, Schrooten C: Imputation of genotypes with low-density chips and its effect on reliability of direct genomic values in Dutch Holstein cattle. J Dairy Sci 2012, 95:876-889.

17. Druet T, Schrooten C, De Roos APW: Imputation of genotypes from different single nucleotide polymorphism panels in dairy cattle. J Dairy Sci 2010, 93:5443-5454.

18. Huang L, Li Y, Singleton AB, Hardy JA, Abecasis G, Rosenberg NA, Scheet $P$ : Genotype-imputation accuracy across worldwide human populations. Am J Hum Genet 2009, 84:235-250.

19. Calus MPL, Veerkamp RF, Mulder HA: Imputation of missing single nucleotide polymorphism genotypes using a multivariate mixed model framework. J Anim Sci 2011, 89:2042-2049.

20. Goddard ME, Hayes BJ: Genomic selection based on dense genotypes inferred from sparse genotypes. Proc Adv Anim Breed Genet 2009, 18:26-29.

21. Gusev A, Shah MJ, Kenny EE, Ramachandran A, Lowe JK, Salit J, Lee CC, Levandowsky EC, Weaver TN, Doan QC, Peckham HE, McLaughlin SF, Lyons MR, Sheth VN, Stoffel M, De La Vega FM, Friedman JM, Breslow JL, Pe'er I: Low-pass genome-wide sequencing and variant inference using identityby-descent in an isolated human population. Genetics 2012, 190:679-689.

22. Zimin AV, Delcher AL, Florea L, Kelley DR, Schatz MC, Puiu D, Hanrahan F, Pertea G, Van Tassell CP, Sonstegard TS, Marçais G, Roberts M, Subramanian $P$, Yorke JA, Salzberg SL: A whole-genome assembly of the domestic cow, Bos taurus. Genome Biol 2009, 10:R42.

23. Cole J: PyPedal: A computer program for pedigree analysis. Computers Electron Agr 2007, 57:107-113.

24. VanRaden PM: Efficient methods to compute genomic predictions. J Dairy Sci 2008 , 91:4414-4423.

25. Hickey JM, Crossa J, Babu R, De los Campos G: Factors affecting the accuracy of genotype imputation in populations from several maize breeding programs. Crop Sci 2012, 52:654

26. Howie B, Fuchsberger C, Stephens M, Marchini J, Abecasis GR: Fast and accurate genotype imputation in genome-wide association studies through pre-phasing. Nat Genet 2012, 44:955-959.

27. Pei Y-F, Li J, Zhang L, Papasian CJ, Deng H-W: Analyses and comparison of accuracy of different genotype imputation methods. PLoS One 2008 3:e3551.

28. Sun C, Wu X-L, Weigel KA, Rosa GJM, Bauck S, Woodward BW, Schnabel RD, Taylor JF, Gianola D: An ensemble-based approach to imputation of moderate-density genotypes for genomic selection with application to Angus cattle. Genet Res 2012, 94:133-150.

29. Browning SR: Missing data imputation and haplotype phase inference for genome-wide association studies. Hum Genet 2008, 124:439-450.

30. Delaneau O, Marchini J, Zagury J-F: A linear complexity phasing method for thousands of genomes. Nat Methods 2011, 9:179-181.

31. Brøndum RF, Ma P, Lund MS, Su G: Short communication: Genotype imputation within and across Nordic cattle breeds. J Dairy Sci 2012, 95:6795-6800

32. Erbe M, Hayes BJ, Matukumalli LK, Goswami S, Bowman PJ, Reich CM, Mason BA, Goddard ME: Improving accuracy of genomic predictions 
within and between dairy cattle breeds with imputed high-density single nucleotide polymorphism panels. J Dairy Sci 2012, 95:4114-4129.

33. Huang Y, Maltecca C, Cassady JP, Alexander LJ, Snelling WM, MacNeil MD: Effects of reduced panel, reference origin, and genetic relationship on imputation of genotypes in Hereford cattle. J Anim Sci 2012, 90:4203-4208.

34. Hayes BJ, Bowman PJ, Daetwyler HD, Kijas JW, Van der Werf JHJ: Accuracy of genotype imputation in sheep breeds. Anim Genet 2012, 43:72-80

35. Stachowicz K, Sargolzaei M, Miglior F, Schenkel FS: Rates of inbreeding and genetic diversity in Canadian Holstein and Jersey cattle. J Dairy Sci 2011, 94:5160-5175.

36. Hinrichs $D$, Thaller $G$ : Pedigree analysis and inbreeding effects on calving traits in large dairy herds in Germany. J Dairy Sci 2011, 94:4726-4733.

37. Zhang Z, Druet T: Marker imputation with low-density marker panels in Dutch Holstein cattle. J Dairy Sci 2010, 93:5487-5494.

38. Dassonneville R, Fritz S, Boichard D, Ducrocq V: Imputation efficiency with different low density chips in French dairy and beef breeds. Interbull Bull 2011, 44:47-50.

39. Dassonneville R, Brøndum RF, Druet T, Fritz S, Guillaume F, Guldbrandtsen B, Lund MS, Ducrocq V, Su G: Effect of imputing markers from a low-density chip on the reliability of genomic breeding values in Holstein populations. J Dairy Sci 2011, 94:3679-3686.

40. Browning SR, Browning BL: Haplotype phasing: existing methods and new developments. Nat Rev Genet 2011, 12:703-714.

41. Johnston J, Kistemaker G, Sullivan PG: Comparison of different imputation methods. Interbull Bull 2011, 44:25-33.

42. Howie B, Marchini J, Stephens M: Genotype imputation with thousands of genomes. G3 2011, 1:457-470.

43. Hickey JM, Kinghorn BP, Tier B, Wilson JF, Dunstan N, Van der Werf JHJ: A combined long-range phasing and long haplotype imputation method to impute phase for SNP genotypes. Genet Sel Evol 2011, 43:12.

44. Daetwyler HD, Wiggans GR, Hayes BJ, Woolliams JA, Goddard ME: Imputation of missing genotypes from sparse to high density using long-range phasing. Genetics 2011, 189:317-327.

45. Hickey JM, Kinghorn BP, Tier B, Werf JH Van D, Cleveland MA: A phasing and imputation method for pedigreed populations that results in a single-stage genomic evaluation. Genet Sel Evol 2012, 44:9.

46. Sargolzaei M, Schenkel FS, Jansen GB, Schaeffer LR: Extent of linkage disequilibrium in Holstein cattle in North America. J Dairy Sci 2008, 91:2106-2117

47. Qanbari S, Pimentel ECG, Tetens J, Thaller G, Lichtner P, Sharifi AR, Simianer $\mathrm{H}$ : The pattern of linkage disequilibrium in German Holstein cattle. Anim Genet 2010, 41:346-356.

48. Bovine HapMap C, Gibbs RA, Taylor JF, Van Tassell CP, Barendse W, Eversole KA, Gill CA, Green RD, Hamernik DL, Kappes SM, Lien S, Matukumalli LK, McEwan JC, Nazareth LV, Schnabel RD, Weinstock GM, Wheeler DA, Ajmone-Marsan P, Boettcher PJ, Caetano AR, Garcia JF, Hanotte O, Mariani P, Skow LC, Sonstegard TS, Williams JL, Diallo B, Hailemariam L, Martinez ML, Morris CA, Silva LO, et al: Genome-wide survey of SNP variation uncovers the genetic structure of cattle breeds. Science 2009, 324:528-532.

49. Corbin LJ, Blott SC, Swinburne JE, Vaudin M, Bishop SC, Woolliams JA: The identification of SNPs with indeterminate positions using the Equine SNP50 BeadChip. Anim Genet 2012, 43:337-339.

50. Bohmanova J, Sargolzaei M, Schenkel FS: Characteristics of linkage disequilibrium in North American Holsteins. BMC Genomics 2010, 11:421.

51. Fadista J, Bendixen C: Genomic position mapping discrepancies of commercial SNP chips. PloS One 2012, 7:e31025.

doi:10.1186/1297-9686-45-3

Cite this article as: Pausch et al:: Imputation of high-density genotypes in the Fleckvieh cattle population. Genetics Selection Evolution 2013 45:3.

\section{Submit your next manuscript to BioMed Central and take full advantage of:}

- Convenient online submission

- Thorough peer review

- No space constraints or color figure charges

- Immediate publication on acceptance

- Inclusion in PubMed, CAS, Scopus and Google Scholar

- Research which is freely available for redistribution

Submit your manuscript at www.biomedcentral.com/submit
C Biomed Central 